Algebraic 85 Geometric Topology

Volume 3 (2003) 147-154

Published: 20 February 2003

\title{
A flat plane that is not the limit of periodic flat planes
}

\author{
DANIEL T. Wise
}

\begin{abstract}
We construct a compact nonpositively curved squared 2-complex whose universal cover contains a flat plane that is not the limit of periodic flat planes.
\end{abstract}

AMS Classification 20F67; 20F06

Keywords CAT(0), periodic flat planes, C(4)-T(4) complexes

\section{Introduction}

Gromov raised the question of which "semi-hyperbolic spaces" have the property that their flats can be approximated by periodic flats $\left[4, \S 6 . \mathrm{B}_{3}\right]$. In this note we construct an example of a compact nonpositively curved squared 2complex $Z$ whose universal cover $\tilde{Z}$ contains an isometrically embedded flat plane that is not the limit of a sequence of periodic flat planes.

A flat plane $\mathbb{E} \hookrightarrow \tilde{Z}$ is periodic if the map $\mathbb{E} \rightarrow Z$ factors as $\mathbb{E} \rightarrow T \rightarrow Z$ where $\mathbb{E} \rightarrow T$ is a covering map of a torus $T$. Equivalently, $\pi_{1} Z$ contains a subgroup isomorphic to $\mathbb{Z} \times \mathbb{Z}$ which stabilizes $\mathbb{E}$ and acts cocompactly on it. A flat plane $f: \mathbb{E} \hookrightarrow \tilde{Z}$ is the limit of periodic flat planes if there is a sequence of periodic flat planes $f_{i}: \mathbb{E} \hookrightarrow \tilde{Z}$ which converge pointwise to $f: \mathbb{E} \rightarrow \tilde{Z}$. In our setting, $\tilde{Z}$ is a 2 -dimensional complex, and so $\mathbb{E} \hookrightarrow \tilde{Z}$ is the limit of periodic flat planes if and only if every compact subcomplex of $\mathbb{E}$ is contained in a periodic flat plane.

In Section 2 we describe a compact nonpositively curved 2-complex $X$ whose universal cover contains a certain aperiodic plane called an "anti-torus". In Section 3 we construct $Z$ from $X$ by strategically gluing tori and cylinders to $X$ so that $\tilde{Z}$ contains a flat plane which is a mixture of the anti-torus and periodic planes. This flat plane is not approximable by periodic flats because it contains a square that does not lie in any periodic flat. Our example $Z$ is a $K(\pi, 1)$ for a negatively curved square of groups, and in Section 4 we describe an interesting related triangle of groups. 

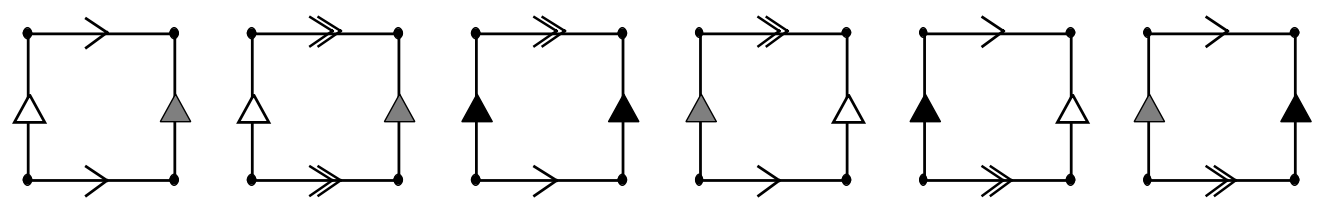

Figure 1: The figure above indicates the gluing pattern for the six squares of $X$. The three vertical edges colored white, grey, and black are denoted $a, b$, and $c$ respectively. The two horizontal edges, single and double arrow, are denoted $x$ and $y$ respectively.

\section{The anti-torus in $X$}

\subsection{The 2-complex $X$}

Let $X$ denote the complex consisting of the six squares indicated in Figure 1. The squares are glued together as indicated by the oriented labels on the edges. Note that $X$ has a unique 0 -cell, and that the notion of vertical and horizontal are preserved by the edge identifications. Let $H$ denote the subcomplex consisting of the 2 horizontal edges, and let $V$ denote the subcomplex consisting of the 3 vertical edges.

The complex $X$, which was first studied in [8], has a number of interesting properties that we record here: The link of the unique 0 -cell in $X$ is a complete bipartite graph. It follows that the universal cover $\widetilde{X}$ is the product of two trees $\tilde{H} \times \tilde{V}$ where $\tilde{H}$ and $\tilde{V}$ are the universal covers of $H$ and $V$. In particular, the link contains no cycle of length $<4$ and so $X$ satisfies the combinatorial nonpositive curvature condition for squared 2 -complexes $[3,1]$ which is a special case of the $C(4)-T(4)$ small-cancellation condition [6].

The 2-complex $X$ was used in [8] to produce the first examples of non-residually finite groups which are fundamental groups of spaces with the above properties. The connection to finite index subgroups arises because while $\widetilde{X}$ is isomorphic to the cartesian product of two trees, $X$ does not have a finite cover which is the product of two graphs.

\subsection{The anti-torus $\Pi$}

The exotic behavior of $X$ can be attributed to the existence of a strangely aperiodic plane $\Pi$ in $\tilde{X}$ that we shall now describe. Let $\tilde{x} \in \widetilde{X}^{0}$ be the basepoint of $\widetilde{X}$. Let $c^{\infty}$ denote the infinite periodic vertical line in $\tilde{X}$ which is the based component of the preimage of the loop labeled by $c$ in $X$. Define $y^{\infty}$ 


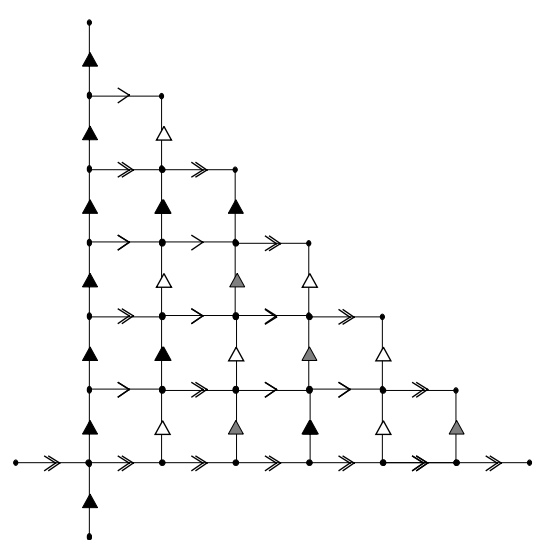

Figure 2: The Anti-Torus $\Pi$ : The plane $\Pi$ above is the convex hull of two periodically labeled lines in $\tilde{X}$. A small region of the northeast quadrant has been tiled by the squares of $X$.

analogously. Let $\Pi$ denote the convex hull in $\tilde{X}$ of the infinite geodesics labeled by $c^{\infty}$ and $y^{\infty}$, so $\Pi=y^{\infty} \times c^{\infty}$. The plane $\Pi$ is tiled by the six orbits of squares in $\tilde{X}$ as in Figure 2. The reader can extend $c^{\infty} \cup y^{\infty}$ to a flat plane by successively adding squares wherever there is a pair of vertical and horizontal edges meeting at a vertex. From a combinatorial point of view, the existence and uniqueness of this extension is guaranteed by the fact that the link of $X$ is a complete bipartite graph.

The "axes" $c^{\infty}$ and $y^{\infty}$ of $\Pi$ are obviously periodic, and using that $X$ is compact, it is easy to verify that for any $n \in \mathbb{N}$, the infinite strips $[-n, n] \times \mathbb{R}$ and $\mathbb{R} \times[-n, n]$ are periodic. However, the period of these infinite strips increases exponentially with $n$. Thus, the entire plane $\Pi$ is aperiodic. Note that to say that $[-n, n] \times \mathbb{R}$ is periodic means that the immersion $([-n, n] \times \mathbb{R}) \rightarrow X$ factors as $([-n, n] \times \mathbb{R}) \rightarrow C \rightarrow X$ where $([-n, n] \times \mathbb{R}) \rightarrow C$ is the universal covering map of a cylinder. The map $\Pi \rightarrow X$ is aperiodic in the sense that it does not factor through an immersed torus.

We conclude this section by giving a brief explanation of the aperiodicity of $\Pi$. A complete proof that $\Pi$ is aperiodic is given in [8]. Let $W_{n}(m)$ denote the word corresponding to the length $n$ horizontal positive path in $\Pi$ beginning at the endpoint of the vertical path $c^{m}$. Thus, $W_{n}(m)$ is the label of the side opposite $y^{n}$ in the rectangle which is the combinatorial convex hull of $y^{n}$ and $c^{m}$. Equivalently, $W_{n}(m)$ occupies the interval $\{m\} \times[0, n]$. For each $n$, the words $\left\{W_{n}(m) \mid 0 \leq m \leq 2^{n}-1\right\}$ are all distinct! Consequently every positive length $n$ word in $x$ and $y$ is $W_{n}(m)$ for some $m$. This implies that the infinite 


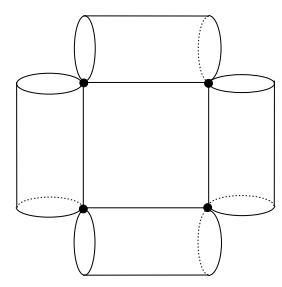

Figure 3: The complex $Y$ is formed by gluing four cylinders to a square.

strip $[0, n] \times \mathbb{R}$ has period $2^{n}$, and in particular $\Pi$ cannot be periodic.

We refer to $\Pi$ as an anti-torus because the aperiodicity of $\Pi$ implies that $c$ and $y$ do not have nonzero powers which commute. Indeed, if $c^{p}$ and $y^{q}$ commuted for $p, q \neq 0$ then the flat torus theorem (see [1]) would imply that $c^{\infty}$ and $y^{\infty}$ meet in a periodic flat plane, which would contradict that $\Pi$ is aperiodic.

\section{The 2-complex $Z$ with a nonapproximable flat}

We first construct a new complex $Y$ as follows: Start with a square $s$, and then attach four cylinders each of which is isomorphic to $S^{1} \times I$. One such cylinder is attached along each side of $s$. The resulting complex $Y$ containing exactly five squares is illustrated in Figure 3.

Let $T^{2}$ denote the torus $S^{1} \times S^{1}$ with the usual product cell structure consisting of one 0 -cell, two 1-cells, and a single square 2 -cell. We let $\widetilde{T}^{2}$ denote the universal cover and we shall identify $\widetilde{T}^{2}$ with $\mathbb{R}^{2}$.

At each corner of $s \subset Y$, there is a pair of intersecting circles in $Y^{1}$, which are boundary circles of distinct cylinders. Note that they meet at an angle of $\frac{3 \pi}{2}$ in $Y$. At each of three (NW, SW, \& SE) corners of $s \subset Y$ we attach a copy of $T^{2}$ by identifying the pair of circles in the 1-skeleton of $T^{2}$ with the pair of intersecting circles noted above at the respective corner of $s$. At the fourth (NE) corner of $s$, we attach a copy of the complex $X$. Here we identify the pair of circles meeting at the corner of $s$ with the pair of perpendicular circles $c$ and $y$ of $X$. We denote the resulting complex by $Z$. Thus, $Z=T^{2} \cup T^{2} \cup T^{2} \cup Y \cup X$. See Figure 4 for a depiction of the 8 squares of $Z-X$ and their gluing patterns.

Definition 3.1 Infinite cross An infinite cross is a squared 2-complex isomorphic to the subcomplex of $\widetilde{T}^{2}$ consisting of $([0,1] \times \mathbb{R}) \cup(\mathbb{R} \times[0,1])$. The base square of the infinite cross is the square $[0,1] \times[0,1]$. 

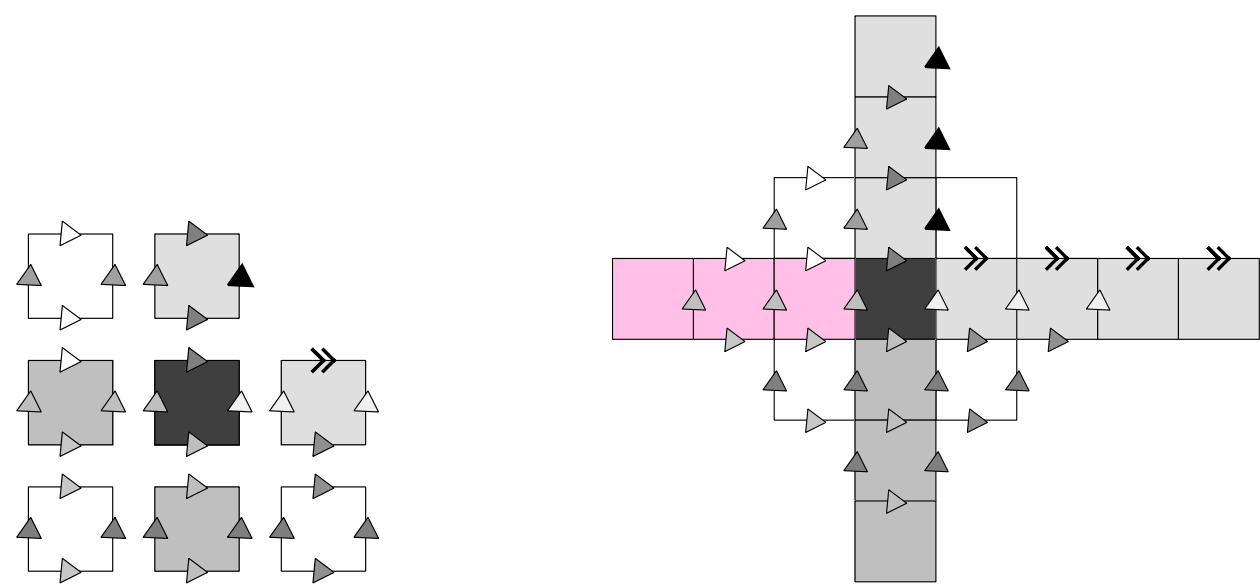

Figure 4: $Z-X$ and $Z$ : The eight squares of the figure on the left are glued together following the gluing pattern to form $Z-X$. To form $Z$, we add a copy of $X$ at the $N E$ corner, identifying the loops in $X$ labeled by $c$ and $y$, with the black single and double arrows of the diagram. The figure on the right represents an infinite cross whose convex hull in $Z$ is not approximable by any periodic plane. Note that while the NW, $\mathrm{SW}$, and SE quarters of this plane are periodic, the NE quarter is an aperiodic quarter of $\Pi$.

The planes containing $s$ : Observe that $Y$ contains various immersions of an infinite cross whose base square maps to $s$. In particular, there are exactly 16 distinct immersed infinite crosses $C \rightarrow Y$ that pass through $s$ exactly once. Each of these infinite crosses extends uniquely to an immersed flat plane in $Z$. Each such flat plane fails to be periodic because its four quarters map to distinct parts of $Z$. Our main result is that these immersed flat planes are not approximable by periodic flat planes because of the following:

Theorem 3.2 (No periodic approximation) There is no immersion of a torus $T^{2} \rightarrow Z$ which contains $s$. Equivalently, there is no periodic plane in $\tilde{Z}$ containing $\tilde{s}$.

Proof We argue by contradiction. Suppose that there is an immersed periodic plane $\Omega$ containing $s$. We shall now produce a rectangle as in Figure 5 that will yield a contradiction. We may assume that a copy of $s$ in $\Omega$ is oriented as in Figure 4. We begin at this copy of $s$ and travel north inside the northern cylinder until we reach another copy $s_{n}$ of $s$. The existence of $s_{n}$ is guaranteed by our assumption that $\Omega$ is periodic. Similarly, we travel east from $s$ to reach a square $s_{e}$. Travelling north from $s_{e}$ and east from $s_{n}$, we trace out the boundary of a rectangle whose NE corner is a square $s_{n e}$ (see Figure 5). 


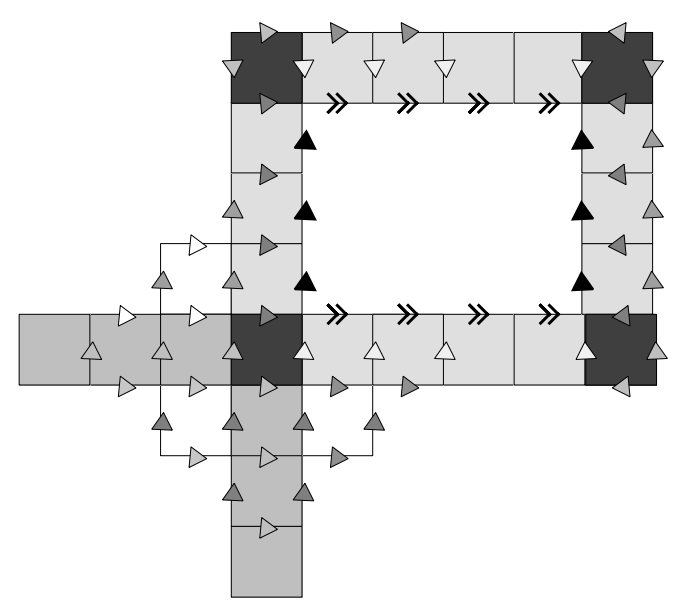

Figure 5: The figure above illustrates one of the four possible contradictions which explain why no periodic plane contains the square $s$.

This yields a contradiction because the inside of this rectangle is tiled by squares in $X$, yet the boundary of this rectangle is a commutator $\left[c^{ \pm n}, y^{ \pm m}\right]$. As explained in Section 2, such a word cannot be trivial in $\pi_{1} X$ because of the anti-torus.

Remark 3.3 Using an argument similar to the above proof, one can show that these sixteen planes are the only flat planes in $\tilde{Z}$ containing $\tilde{s}$. One considers the pair of "axes" intersecting at $\tilde{s}$ in a plane containing $\tilde{s}$. If this plane is different from each of the 16 mentioned above, then some translate of $\tilde{s}$ must appear along one of these "axes". The infinite strip in the plane whose corners are these two $s$ squares yields a contradiction similar to the one obtained above.

Remark 3.4 While $X$ is a rather pathological complex, we note that every flat plane in $\tilde{X}$ is the limit of periodic flat planes. Indeed this holds for any compact 2-complex $X$ whose universal cover is isomorphic to the product of two trees [8].

\section{Polygons of groups}

\subsection{The algebraic angle versus the geometric angle}

Since the elements $c$ and $y$ have axes which intersect perpendicularly in a plane in $\tilde{X}$, the natural geometric angle between the subgroups $\langle c\rangle$ and $\langle y\rangle$ 
of $\pi_{1} X$ is $\frac{\pi}{2}$. However, the algebraic Gersten-Stallings angle (see [7]) between these subgroups is $\leq \frac{\pi}{3}$. To see this, we must show that there is no non-trivial relation of the form $c^{k} y^{l} c^{m} y^{n}=1$.

Since $\tilde{X}$ is isomorphic to the cartesian product $\tilde{V} \times \tilde{H}$, of two trees and $c$ and $y$ correspond to distinct factors, it follows that the only relations that must be checked are rectangular (i.e., $|k|=|m|$ and $|l|=|n|$ ). However, these are easily ruled out by the anti-torus $\Pi$ and the fact that $X$ is nonpositively curved.

\subsection{Square of groups and triangle of groups}

The complex $Z$ can be thought of in a natural way as a $K(\pi, 1)$ for a negatively curved square of groups (see $[7,5,2]$ ) with cyclic edge groups and trivial face group.

Because the algebraic angle between $\langle c\rangle$ and $\langle y\rangle$ in $\pi_{1} X$ is $\leq \frac{\pi}{3}$, it is tempting to form an analogous nonpositively curved triangle of groups $D$. The face group of $D$ is trivial, the edge groups of $D$ are cyclic, the vertex groups of $D$ are isomorphic to $\pi_{1} X$, and each edge group of $D$ is embedded on one (clockwise) side as $\langle c\rangle$ and on the other (counter-clockwise) side as $\langle y\rangle$. This can be done so that the resulting triangle of groups $D$ has $\mathbb{Z}_{3}$ symmetry. The tension between the algebraic and geometric angles should endow $\pi_{1} D$ with some interesting properties. For instance, I suspect that $\pi_{1} D$ fails to be the fundamental group of a compact nonpositively curved space, but it fails for reasons different from the usual types of problems.

Acknowledgments I am grateful to the referee for the helpful corrections. This research was supported by an NSERC grant.

\section{References}

[1] Martin R Bridson, André Haefliger, Metric spaces of non-positive curvature, Springer-Verlag, Berlin (1999)

[2] Jon Michael Corson, Complexes of groups, Proc. London Math. Soc. (3) 65 (1992) 199-224

[3] M Gromov, Hyperbolic groups, from: "Essays in group theory", Math. Sci. Res. Inst. Publ. 8, Springer, New York (1987) 75-263

[4] M Gromov, Asymptotic invariants of infinite groups, from: "Geometric group theory, Vol. 2 (Sussex, 1991)", Cambridge Univ. Press, Cambridge (1993) 1-295 
[5] André Haefliger, Complexes of groups and orbihedra, from: "Group theory from a geometrical viewpoint (Trieste, 1990)", (É Ghys, A Haefliger, A Verjovsky, editors), World Sci. Publishing, River Edge, NJ (1991) 504-540

[6] Roger C Lyndon, Paul E Schupp, Combinatorial group theory, SpringerVerlag, Berlin (1977), ergebnisse der Mathematik und ihrer Grenzgebiete, Band 89

[7] John R Stallings, Non-positively curved triangles of groups, from: "Group theory from a geometrical viewpoint (Trieste, 1990)", (É Ghys, A Haefliger, A Verjovsky, editors), World Sci. Publishing, River Edge, NJ (1991) 491-503

[8] Daniel T Wise, Non-positively curved squared complexes, aperiodic tilings, and non-residually finite groups, Ph.D. thesis, Princeton University (1996)

Department of Mathematics and Statistics, McGill University

Montreal, Quebec H3A 2K6, Canada

Email: wise@math.mcgill.ca

Received: 27 January 2003 